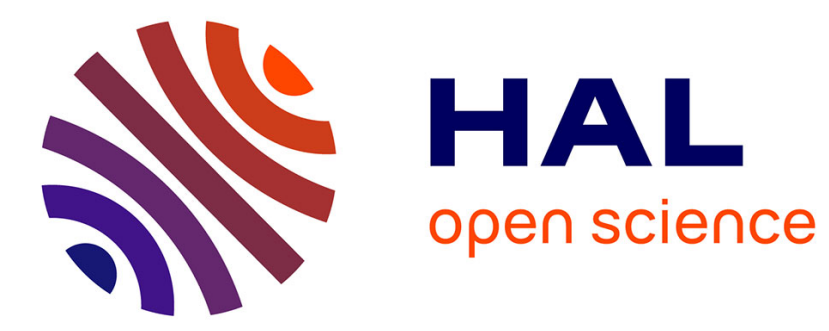

\title{
Optimized sub-wavelength grating mirror design for mid-infrared wavelength range
}

Christyves Chevallier, Nicolas Fressengeas, Frédéric Genty, Joël Jacquet

\section{To cite this version:}

Christyves Chevallier, Nicolas Fressengeas, Frédéric Genty, Joël Jacquet. Optimized sub-wavelength grating mirror design for mid-infrared wavelength range. Applied physics. A, Materials science \& processing, 2011, 103 (4), pp.1139-1144. 10.1007/s00339-010-6059-4 . hal-00521032

\section{HAL Id: hal-00521032 \\ https://hal-centralesupelec.archives-ouvertes.fr/hal-00521032}

Submitted on 24 Sep 2010

HAL is a multi-disciplinary open access archive for the deposit and dissemination of scientific research documents, whether they are published or not. The documents may come from teaching and research institutions in France or abroad, or from public or private research centers.
L'archive ouverte pluridisciplinaire HAL, est destinée au dépôt et à la diffusion de documents scientifiques de niveau recherche, publiés ou non, émanant des établissements d'enseignement et de recherche français ou étrangers, des laboratoires publics ou privés. 


\title{
Optimized sub-wavelength grating mirror design for mid infrared
}

\section{wavelength range}

\author{
C. Chevallier ${ }^{\star}$, N. Fressengeas, F. Genty, J. Jacquet
}

Laboratoire Matériaux Optiques, Photonique et Systèmes, 2 rue Edouard Belin 57070 Metz, France, Unité de Recherche Commune à l’Université Paul Verlaine - Metz et Supélec

Received: 23/09/2010 / Revised version: date

\begin{abstract}
Several designs of sub-wavelength grating mirrors adapted to mid-infrared operation are reported with several percents of tolerance for the grating fabrication. These designs have been automatically optimized by the use of a genetic-based algorithm to maximize a quality factor defined to meet the requirements of a VCSEL cavity mirror. These mirrors are devoted to an integration in VCSEL operating near $\lambda=2.3 \mu \mathrm{m}$, with a large bandwidth, very high reflectivity coefficient for transverse magnetic mode only, polarization selectivity and a thickness as low as $2 \mu \mathrm{m}$.
\end{abstract}

\section{Introduction}

The existence of strong absorption lines for polluting gas $\left(\mathrm{CH}_{4}, \mathrm{CO}_{2}, \mathrm{CO}, \ldots\right)$ in the mid-infrared (MIR) wavelength range beyond $2 \mu \mathrm{m}$ presents a great interest for

\footnotetext{
* Corresponding author: christyves.chevallier@supelec.fr
}

spectroscopic measurements. Due to their particular properties, VCSELs appear as stable, compact and very efficient light sources for such an application [1,2]. Microcavity VCSELs traditionally include two distributed Bragg mirrors (DBR) which can be fabricated with semiconductor or dielectric materials. In this wavelength range, due to a relatively low index contrast $(\Delta n \sim 0.5)$, one semiconductor DBR must contain at least 20 pairs of quarter-wavelength layers to reach minimum reflectivity $(R>99 \%)$ required for Room Temperature (RT) laser operation [3]. Moreover, such reflectors usually provide bandwidths as large as $300 \mathrm{~nm}$ [3] but are polarization independent which can alter the stability of emitted light by brutal polarization switches and mode competitions [4]. Furthermore, the thickness of such DBR mirrors increases with the wavelength. Since the number of layers for one DBR has to be larger than 40 to keep a high enough reflectivity and a 300 nm-large bandwidth, 
in the MIR range, the total thickness of VCSELs can become as high as $15 \mu \mathrm{m}$ for an operating wavelength of $\lambda=2.5 \mu \mathrm{m}[5]$ which impairs the electro-thermo-optical stability of the device.

For $\lambda \geq 2.3 \mu \mathrm{m}$, the best performances were obtained with devices developed from the AlGaInAsSb semiconductor material system. Two different electrically pumped VCSEL structures, an all-epitaxial monolithic microcavity and an hybrid one, have been recently successfully developed for an emission at $\lambda \geq 2.3 \mu \mathrm{m}$. The all-epitaxial monolithic structure [6] is made of two Te-doped AlAsSb/ GaSb DBRs and an $\mathrm{n}^{++} \operatorname{InAs}(\mathrm{Sb}) / \mathrm{p}^{++} \mathrm{GaSb}$ tunnel junction. This laser structure operates at RT in a CW mode near $2.3 \mu \mathrm{m}[5]$ and in quasi-CW $(5 \%, 1 \mu \mathrm{s})$ up to $2.63 \mu \mathrm{m}[7]$. The hybrid structure contains a top dielectric mirror and a buried tunnel junction and operates in CW emission at RT in the $2.4 \mu \mathrm{m}-2.6 \mu \mathrm{m}$ wavelength range [8]. However, due to the abscence of polarization selectivity in the structure, both of these VCSELs are likely to present hopes during laser operation.

One solution to provide a polarization selectivity is the replacement of the top cavity DBR by a sub-wavelength grating mirror (SGM) $[9,10]$. Furthermore, a SGM with a low index sublayer which increases the reflectivity of the grating [11] is thiner than a DBR for an equivalently wide spectrum thus providing better electro-thermo-optical stability. Moreover, owing to its one dimensional symmetry, such a mirror provides a polarization selectivity of the reflected light. Thus, with both these qualities, the integration of a SGM in mid-IR operating VCSELs appears as very promising to improve the beam quality and increase the wavelength of emission of such devices.

Such mirrors operate by excitation and reflection of transverse modes which propagate in the grating direction [12]. Their reflectivity is not straightforward [13] and must be numerically computed by methods such as Rigorous Coupled-Wave Analysis (RCWA) [14] or FiniteDifference Time-Domain (FDTD) [15]. This is the reason why the design of the SGM characteristic lengths is still an important issue [11].

In this paper, an automated optimized design of SGM mirrors for applications in the mid-IR wavelength range is proposed. The optimization is performed by the carefully definition of a quality factor which reflects the characteristics that a SGM has to meet for efficient integration in VCSEL structures. This quality factor is then optimized thanks to a genetic algorithm $[16,17]$. In the following, the section 2 is devoted to the definition of the quality factor and the means used for its maximization, while the section 3 presents two different designs of $\mathrm{Si} / \mathrm{SiO}_{2}$ SGMs obtained with the previously described method and assesses their predicted performances. The two designs, with and without the use of a low index layer below SGM, have been optimized to have the largest bandwidth with a $99.9 \%$ high reflectivity centered at $\lambda=2.3 \mu \mathrm{m}$. 


\section{Designing and optimization of a sub-wavelength grating mirror}

Reflectors designed in this work have been optimized as VCSEL top-mirrors which require high reflectivity for the widest possible bandwidth. The bandwidth was defined as the wavelength range for which the transverse magnetic mode (TM) has a reflection coefficient $R_{T M}$ higher than $99.9 \%$. A $99.5 \%$ value would surely have been enough to reach the laser threshold, but the remaining $0.4 \%$ was kept as a security margin to account for possible experimental growth imperfections.

In order to prevent mode hopping, the mirror must also be polarization selective. The reflection coefficient of the transverse electric mode $R_{T E}$ was therefore set to reach a maximum value of $90 \%$ over the whole bandwidth, so as to assure the device to lase only in the TM mode. The bandwidth has also been centered at $\lambda_{0}$ where the VCSEL structure has been optimized.

In addition to these requirements, the mirror optimization is made under technological constraints. The first one is the photolithography resolution which limits the size of the filled and etched lengths of the grating $\left(L_{f}\right.$ and $L_{e}$ on Figure 1). A minimum value of $500 \mathrm{~nm}$ was set for each one so as to ease the lithography process. The second limitation is the shape factor $S F$ defined as the etched length $L_{e}$ to grating thickness $T_{g}$ ratio, which is kept at a minimum value of 0.9 . This means that the

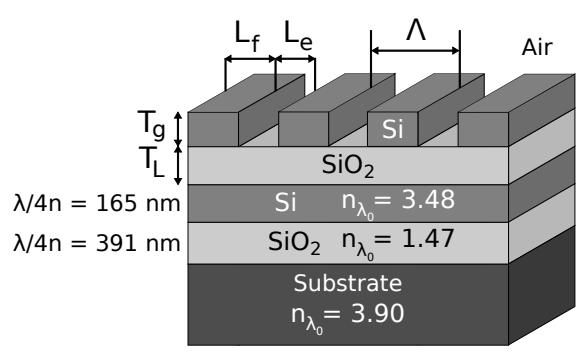

Fig. 1: Scheme of the reflector. A sub-wavelength grating mirror is combined with two quarter-wave layers to reach $99.9 \%$ reflectivity. The substrate represents the VCSEL's phase match layer. The etched length $L_{e}$, filled length $L_{f}$ and thickness $T_{g}$ of the grating and the sublayer thickness $T_{L}$ are the free parameters which are searched to optimize the SGM for VCSEL operation.

optimization retains only the designs with wide grooves since a squared pattern is easier to etch than a deep one.

The simulation of the device is computed in Sage [18] by a stable Rigorous Coupled-Wave Analysis software [14, 19] (RCWA) which finds an exact solution of Maxwell's equations for the electromagnetic diffraction problem. It is thus possible to compute the reflection efficiency of any periodic structure at the 0 -order of diffraction and to numerically evaluate reflection spectra for both TM and TE mode in the mid-infrared wavelength range.

A numerical analysis of such a computed numerical spectrum can be made to quantitatively assess the mirror performances according to the above mentionned criteria. We thus propose to define the quality factor $Q$ as follows : 


$$
Q=\frac{\Delta \lambda}{\lambda_{0}} \frac{1}{N} \sum_{\lambda=\lambda_{1}}^{\lambda_{2}} R_{T M}(\lambda) g(\lambda)
$$

The polarization selectivity of the grating is set by selecting the range of wavelengths $\lambda$ around $\lambda_{0}$ for which $R_{T M}$ is higher than $99.9 \%$ and $R_{T E}$ is lower than $90 \%$. The centering of the bandwidth around $\lambda_{0}$ is made by performing a gaussian weighted average of the $R_{T M}$ values on the $N$ points of the bandwidth $\left[\lambda_{1}, \lambda_{2}\right]$. The largest possible bandwidth is obtained by multiplying the latter average by the bandwidth itself $\Delta \lambda=\left|\lambda_{2}-\lambda_{1}\right|$ in order to increase the weight of large bandwidth designs during the optimization. $Q$ is finally normalized by $\lambda_{0}$.

The definition of the quality factor $Q$ representing the performance of the mirror allows the use of an optimization algorithm to automatically search for the best design [20]. The lengths $L_{e}, L_{f}$, the grating thickness $T_{g}$ and the sublayer thickness $T_{L}$ are the parameters of the design which are adjusted to maximize the quality. As can be seen by performing Newton-like optimization on $Q$, this optimization problem has many local maxima. Such a multimodality requires a robust and, above all, global optimization method. Genetic algorithms $[16,21]$ are well suited for this kind of problem since they search from a set of points which evolves from probabilistic transition rules to find an optimal solution. The use of a population of points instead of a single point decreases the chance to find a local maximum by exploring simultaneously many peaks. Another important asset of this method is the fact that no derivability condition has to be set on $Q$ [17]. This is particularly interesting here since $Q$ is not assured to be derivable everywhere against all variables. Moreover genetic algorithms use randomized but not directionless search leading to statistically promote the largest peaks of the $Q$ function which represent the most tolerant designs, which is one goal of our optimization.

With the same definition of the quality factor $Q$, it is possible to calculate the tolerances on each dimension of the mirror. Tolerance is computed by varying one parameter at the time while keeping the other ones constant. For each value of this parameter, spectra are computed and the normalized bandwidth $\Delta \lambda / \lambda_{0}$ versus the length of the parameter can be plotted. The range where the mirror meets VCSEL requirements defines the interval of tolerance of the parameter, it corresponds to a nonzero value for the normalized bandwidth, i.e. when $R_{T M}$ becomes higher than $99.9 \%$ with $R_{T E}$ lower than $90 \%$ at $\lambda_{0}$.

The first optimizations of silicium ( $n=3.475)$ subwavelength grating mirror with a silica $(n=1.47)$ sublayer on a GaSb substrate $(n=3.9)$ show us too narrow bandwidths of about $20 \mathrm{~nm}$ (Figure 2a) because of the optimization constraints we have set as explained above. A pair of $\mathrm{Si} / \mathrm{SiO}_{2}$ quarter-wave layer as in a DBR has been added below the SGM to increase the bandwidth and the reflectivity above $99.9 \%$ (Figure $2 \mathrm{~b}$ ). Since this type of mirror is not polarization dependent, only one pair is used, allowing to keep the reflectivity of the TE 


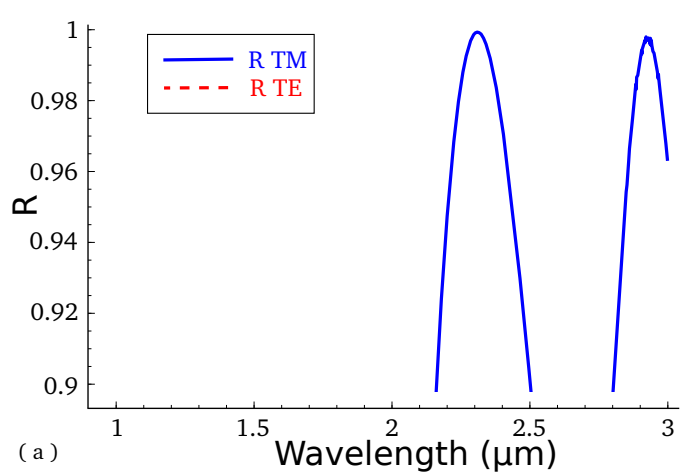

(a) SGM without quarter-wave pair

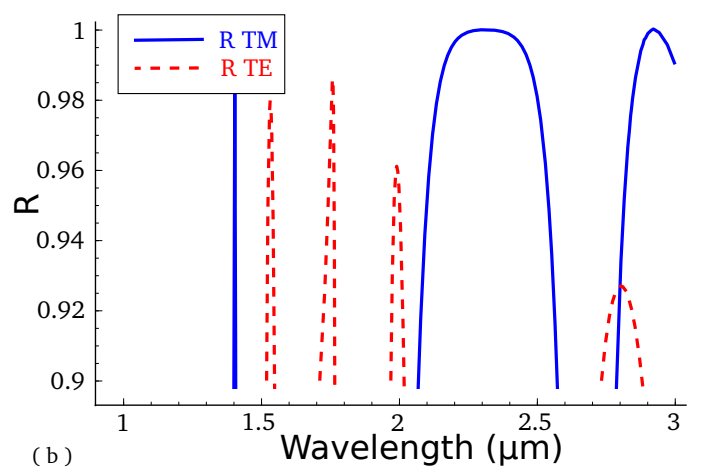

(b) SGM with quarter-wave pair

Fig. 2: Reflection spectra of TM (solid blue) and TE (dashed red) mode.

mode at $\lambda_{0}$ below the $90 \%$ threshold. With this structure, two different designs have been optimized, the first one has a silica low index sub-layer below the grating whereas the second one has a silicium sub-layer as a phase match layer between the grating and the quarterwave pair.

\section{Optimized mirrors centered at $2.3 \mu \mathrm{m}$}

\subsection{Sub-wavelength grating mirror with a silica} sublayer.

The reflector presented on Figure 1 is a combination of a subwavelength grating of silicium and two quarter-wave layers of silicium and silica which increase the reflectivity to very high values of $99.9 \%$. The SGM is on top of a silica low index layer as described in [11]. The choice of silicium is made in regard to its well known properties and ease to process. The substrate corresponds to the GaSb match layer of the final VCSEL with an optical index of 3.9. During the optimization, the pattern

Table 1: Lengths and tolerances of the optimized mirror with a silica sublayer.

Optimum Tolerances for $R_{T M}>99.9 \%$

$\begin{array}{lll}T_{g} & 0.715 \mu \mathrm{m} & 0.678 \mu \mathrm{m}<T_{g}<0.745 \mu \mathrm{m} \\ T_{L} & 0.017 \mu \mathrm{m} & 0 \mu \mathrm{m}<T_{L}<0.090 \mu \mathrm{m} \\ \Lambda & 1.304 \mu \mathrm{m} & 1.028 \mu \mathrm{m}<\Lambda<1.436 \mu \mathrm{m} \\ L_{f} / \Lambda & 48 \% & 42 \%<L_{f} / \Lambda<51 \%\end{array}$

sizes $L_{e}$ and $L_{f}$ are allowed to vary between $0.5 \mu \mathrm{m}$ and $1 \mu \mathrm{m}$ while thicknesses $T_{g}$ and $T_{L}$ are only limited below $1 \mu \mathrm{m}$. These are severe constraints for the optimization algorithm which would rather lead to thiner patterns of less than $260 \mathrm{~nm}$ for a small shape factor $S F$ of less than $0.4[11]$

The optimization returns a design (Table 1) with a grating thickness $T_{g}$ of $0.715 \mu \mathrm{m}$ for a filled length $L_{f}$ of $0.629 \mu \mathrm{m}$ and an etched length $L_{e}$ of $0.675 \mu \mathrm{m}$. This design respects the technological constraints whith patterns longer than $0.500 \mu \mathrm{m}$ and a shape factor $S F$ of 


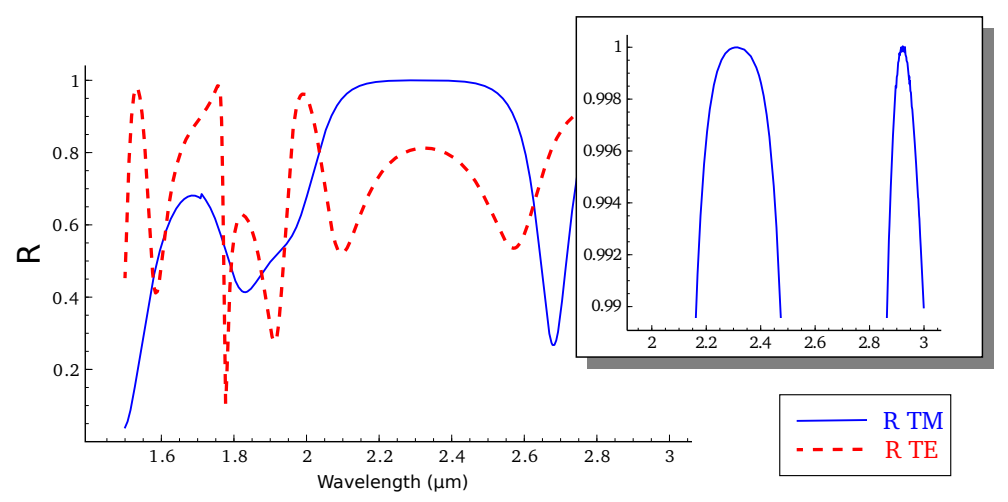

Fig. 3: Reflection spectra of TM (solid blue) and TE (dashed red) mode of the silica sublayer mirror with a $152 \mathrm{~nm}$ bandwidth.

0.94. The silica sublayer has a thickness $T_{L}$ of only $17 \mathrm{~nm}$ but this length has the best tolerance and doesn't significantly affect the quality of the mirror while it is kept thiner than $90 \mathrm{~nm}$. The spectra of this mirror (Figure 3) show us a $152 \mathrm{~nm}$ bandwidth mirror $\left(\Delta \lambda / \lambda_{0}=6.6 \%\right)$ centered at $2.315 \mu \mathrm{m}$. The $R_{T M}$ coefficient reaches very high values above $99.9 \%$ whereas $R_{T E}$ is kept below $80 \%$. Such a mirror satisfies all of our requirements for a VCSEL application.

This design gives a very high reflective and polarized mirror with a large bandwidth. In addition to these qualities, the optimization found a very tolerant design with between $4 \%$ and $15 \%$ of error allowed on each dimension.

However, it can be noticed that the optimization suggests that the silica sublayer be as thin as possible. Therefore, and though it impairs the use of a selective etching process, we have tried our optimization method on a second design from which the silica sublayer has been removed (Figure 4a).

Table 2: Lengths and tolerances of the optimized mirror with a silicium sublayer.

Optimum Tolerances for $R_{T M}>99.9 \%$

$\begin{array}{llll}T_{g} & 0.700 \mu \mathrm{m} & 0.679 \mu \mathrm{m}<T_{g}<0.741 \mu \mathrm{m} & (4.4 \%) \\ T_{L} & 0.201 \mu \mathrm{m} & 0.119 \mu \mathrm{m}<T_{L}<0.256 \mu \mathrm{m} & (34 \%) \\ \Lambda & 1.236 \mu \mathrm{m} & 1.037 \mu \mathrm{m}<\Lambda<1.500 \mu \mathrm{m} & (19 \%) \\ L_{f} / \Lambda & 49 \% & 47 \%<L_{f} / \Lambda<53 \%\end{array}$

\subsection{Sub-wavelength grating mirror with a silicium}

sublayer.

This leads to a design where the silica sublayer is replaced by a silicium one as a phase match layer between the grating and the quarter-wave layers. The optimization is performed in the same conditions and results in a $0.700 \mu \mathrm{m}$ thick grating with a $0.635 \mu \mathrm{m}$ etched length $L_{e}$ and $0.601 \mu \mathrm{m}$ filled length $L_{f}$ (Table 2). The thickness of the silicium sublayer is $0.201 \mu \mathrm{m}$. This design has higher tolerance values up to $34 \%$ and a larger bandwidth of $206 \mathrm{~nm}\left(\Delta \lambda / \lambda_{0}=9 \%\right)$ centered at $\lambda_{0}=2.303 \mu \mathrm{m}$ as shown on Figure 4b. 


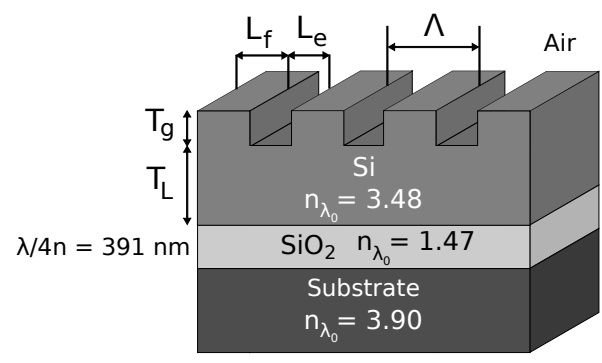

(a) Scheme of the mirror with silicium as a phase match layer between the grating and the quarter-wave layer.

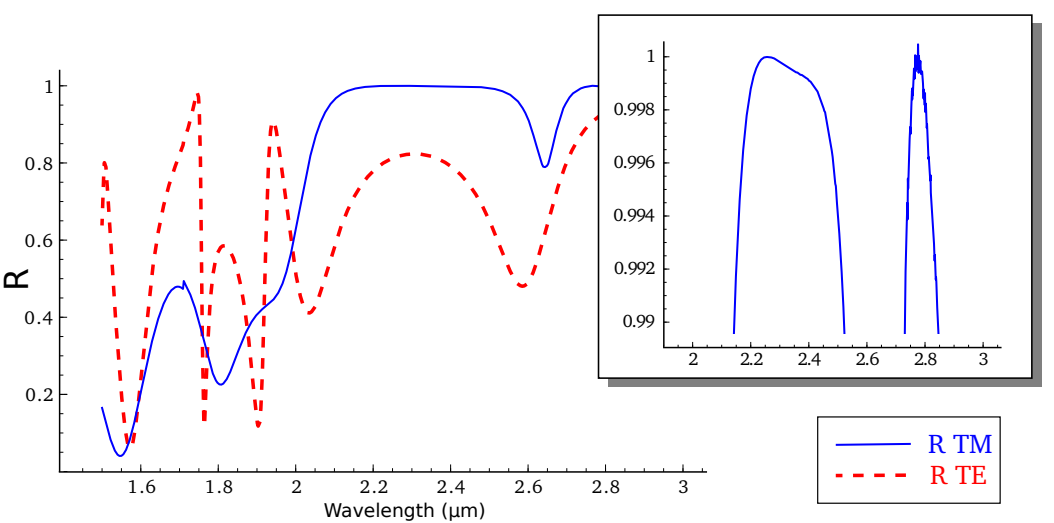

(b) Reflection spectra of TM (solid blue) and TE (dashed red) mode with a $206 \mathrm{~nm}$ bandwidth.

Fig. 4: Second design with a silicium sublayer.

Despite higher tolerances and the absence of nanometric sublayer, this second mirror requires to precisely control the etching process since the shape factor is smaller $(S F=0.9)$ and no selective etching method can be used.

\subsection{Influence of the grating period on the quality of the} mirrors

The computation of the tolerance allows the study of the influence of parameters of the design on the mirror quality. We have chosen to present the influence of the grating period which highlights two different phenomena. On Figure 5 , the normalized bandwidth $\Delta \lambda / \lambda_{0}$ is plotted versus the grating period $\Lambda$ for $R_{T M}$ thresholds of $99.9 \%$ and $99.99 \%$. The grating period varies from $1 \mu \mathrm{m}$ to $1.5 \mu \mathrm{m}$ which is the range for which the SGM has a $99.9 \%$ high bandwidth. The others parameters of the design are kept at their optimal values (Table 1 and 2). The arrow on the graphs points the optimized grating period presented previously.

For both designs, the quality of the mirror increases for periods shorter than the optimal one. The mirror with a silica sublayer presents a bandwidth (Figure 5a) as large as $335 \mathrm{~nm}$ for a $1.068 \mu \mathrm{m}$ period $\left(\Delta \lambda / \lambda_{0}=14.9 \%\right)$ which is more than twice the values found by the optimization. The reason why such designs are not retained by the optimization algorithm is that they have smaller etched lengths, here $L_{e}=0.553 \mu \mathrm{m}$, and since the grating thickness $T_{g}$ is kept at its optimal value of $0.715 \mu \mathrm{m}$, the shape factor $S F$ becomes as low as 0.77 , which is forbidden by our constraints. This confirms that constraints are severe and more performant designs can be found with a smaller shape factor [11].

The second phenomena is that the bandwidth becomes higher than $99.99 \%$ for longer periods. Figure $5 \mathrm{~b}$ 


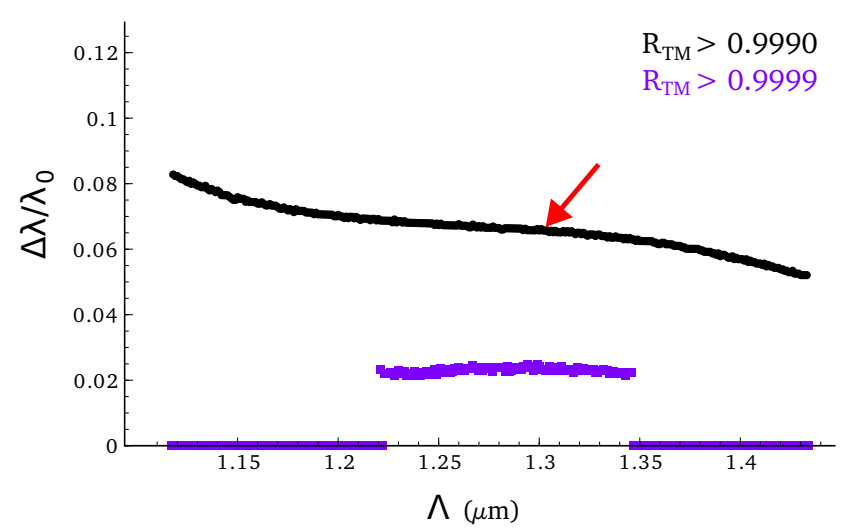

(a) SGM with a $\mathrm{SiO}_{2}$ sublayer

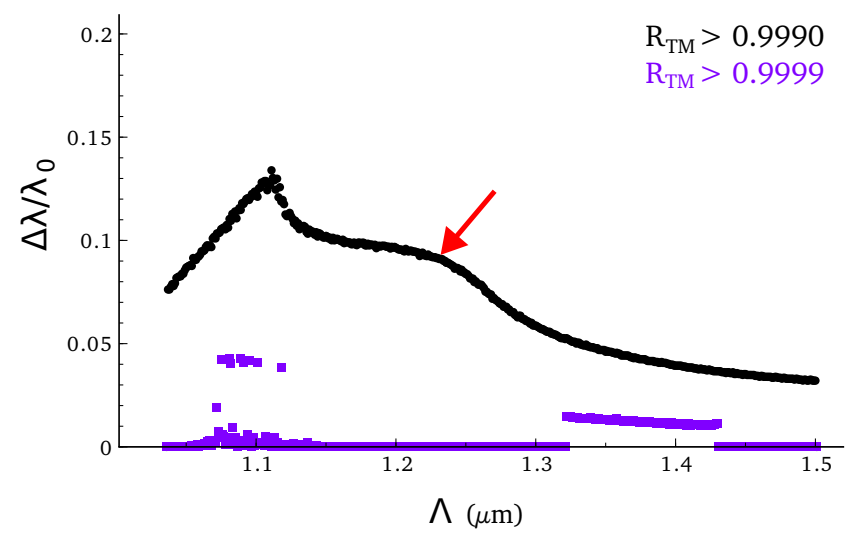

(b) SGM with a Silicium sublayer

Fig. 5: Tolerance of the grating period : normalized bandwidth $\Delta \lambda / \lambda_{0}$ vs. grating period $\Lambda$ for a $R_{T M}$ threshold of $99.9 \%$ (black) and $99.99 \%$ (purple). The arrow indicates the design found by the optimization algorithm.

exhibits a $30 \mathrm{~nm}$ bandwidth for a $1.322 \mu \mathrm{m}$ period SGM with a silicium sublayer for $R_{T M}>99.99 \%$. This shows that the bandwidth can reach higher reflective values at $\lambda_{0}$ with an adjusted design, but for a narrower bandwidth since its width at $R_{T M}=99.9 \%$ is only of $121 \mathrm{~nm}$ which is $80 \mathrm{~nm}$ less than the optimal value. This point demonstrates that our program really searchs for the widest possible bandwidth for a precise $R_{T M}$ value.

\section{Conclusion}

In this paper, two SGM mirrors as thin as $2 \mu \mathrm{m}$ and exhibiting the required properties for a VCSEL integration at $\lambda=2.3 \mu \mathrm{m}$ were presented. These mirrors combine a large bandwidth of more than $150 \mathrm{~nm}$ with a polarization selectivity by keeping $R_{T E}$ below 0.8 in the wavelength range where the VCSEL would operate. With the definition of a quality factor of VCSEL mirrors, the design optimization process is automated and can be eas- ily performed under technological constraints. This factor also allows the computation of tolerances which are higher than 4 percents on the different lengths for which the mirrors should be easy to produce. The integration of such SGM mirrors in mid-IR emitting VCSELs would improve their emission properties allowing the fabrication of CW operating devices above $2.3 \mu \mathrm{m}$.

\section{Acknowledgments}

The authors gratefully acknowledge the financial support by the French ANR (Project Marsupilami, contract ANR-09-BLAN-0166-03). This work was also partly funded by the InterCell grant (http://intercell.metz.supelec.fr) by INRIA and Région Lorraine (CPER2007).

\section{References}

1. Andreas Hangauer, Jia Chen, Rainer Strzoda, Markus Ortsiefer, and Markus-Christian Amann. Wavelength 
modulation spectroscopy with a widely tunable InPbased $2.3 \mu \mathrm{m}$ vertical-cavity surface-emitting laser. Opt. Lett., 33(14):1566-1568, July 2008.

2. J. Chen, A. Hangauer, R. Strzoda, and M. C. Amann. VCSEL-based calibration-free carbon monoxide sensor at $2.3 \mu \mathrm{m}$ with in-line reference cell. Applied Physics B: Lasers and Optics, April 2010.

3. A. Perona, A. Garnache, L. Cerutti, A. Ducanchez, S. Mihindou, P. Grech, G. Boissier, and F. Genty. $\mathrm{AlAsSb} / \mathrm{GaSb}$ doped distributed bragg reflectors for electrically pumped VCSELs emitting around $2.3 \mu \mathrm{m}$. Semiconductor Science and Technology, 22(10):1140-1144, October 2007.

4. A. Ouvrard, A. Garnache, L. Cerutti, F. Genty, and D. Romanini. Single-frequency tunable sb-based VCSELs emitting at $2.3 \mu \mathrm{m}$. IEEE Photonics Technology Letters, 17(10):2020-2022, October 2005.

5. L. Cerutti, A. Ducanchez, G. Narcy, P. Grech, G. Boissier, A. Garnache, E. Tournié, and F. Genty. GaSb-based VCSELs emitting in the mid-infrared wavelength range $(2-3 \mu \mathrm{m})$ grown by MBE. Journal of Crystal Growth, 311(7):1912-1916, 2009.

6. L. Cerutti, A. Ducanchez, P. Grech, A. Garnache, and F. Genty. Room-temperature, monolithic, electricallypumped type-l quantum-well Sb-based VCSELs emitting at $2.3 \mu \mathrm{m}$. Electronics Letters, 44(3):203-205, 2008.

7. A. Ducanchez, L. Cerutti, P. Grech, F. Genty, and E. Tournié. Mid-infrared GaSb-based EP-VCSEL emitting at $2.63 \mu \mathrm{m}$. Electronics Letters, 45(5):265-267, 2009.

8. Alexander Bachmann, Shamsul Arafin, and Kaveh Kashani-Shirazi. Single-mode electrically pumped GaSb- based VCSELs emitting continuous-wave at 2.4 and 2.6 $\mu \mathrm{m}$. New Journal of Physics, 11(12):125014, 2009.

9. M.C.Y. Huang, Y. Zhou, and C.J. Chang-Hasnain. A surface-emitting laser incorporating a high-indexcontrast subwavelength grating. Nat. Photon., 1(2):119122,2007 .

10. I. Chung-Sung, J. Mork, P. Gilet, and A. Chelnokov. Subwavelength grating-mirror VCSEL with a thin oxide gap. IEEE Photonics Technology Letters, 20:105-107, January 2008.

11. C.F.R. Mateus, M.C.Y. Huang, Y. Deng, A.R. Neureuther, and C.J. Chang-Hasnain. Ultrabroadband mirror using low-index cladded subwavelength grating. IEEE Photon. Technol. Lett., 16(2):518-520, 2004.

12. Frank Brückner, Daniel Friedrich, Tina Clausnitzer, Michael Britzger, Oliver Burmeister, Karsten Danzmann, Ernst B. Kley, Andreas Tünnermann, and Roman Schnabel. Realization of a monolithic high-reflectivity cavity mirror from a single silicon crystal. Physical Review Letters, 104(16):163903+, Apr 2010.

13. Robert Magnusson and Mehrdad Shokooh-Saremi. Physical basis for wideband resonant reflectors. Opt. Express, 16(5):3456-3462, 2008.

14. M. G. Moharam, Drew A. Pommet, Eric B. Grann, and T. K. Gaylord. Stable implementation of the rigorous coupled-wave analysis for surface-relief gratings: enhanced transmittance matrix approach. J. Opt. Soc. Am. A, 12(5):1077-1086, 1995.

15. K. Yee. Numerical solution of initial boundary value problems involving maxwell's equations in isotropic media. IEEE Trans. on Antennas and Propagat., 14(3):302- 
307, May 1966.

16. Donald Goodman.

Galileo $\quad 1.0 \mathrm{~b}$ http://sourceforge.net/projects/galileo/, February 2003

17. David E. Goldberg. Genetic Algorithms in Search, Optimization and Machine Learning. Addison-Wesley Longman Publishing Co., Inc., Boston, MA, USA, 1989.

18. William A. Stein. Sage mathematics software (version 4.3). http://www.sagemath.org/, February 2010. The Sage Group

19. Helmut Rathgen. mrcwa 20080820 http://mrcwa.sourceforge.net/, February 2010.

20. Jianpeng Wang, Yunxia Jin, Jianda Shao, and Zhengxiu Fan. Optimization design of an ultrabroadband, highefficiency, all-dielectric grating. Opt. Lett., 35(2):187189, January 2010.

21. Dmitrey L. Kroshko. OpenOpt 0.27 . http://openopt.org/, December 2009.

1 Scheme of the reflector. A sub-wavelength grating mirror is combined with two quarterwave layers to reach $99.9 \%$ reflectivity. The substrate represents the VCSEL's phase match layer. The etched length $L_{e}$, filled length $L_{f}$ and thickness $T_{g}$ of the grating and the sublayer thickness $T_{L}$ are the free parameters which are searched to optimize the SGM for VCSEL operation. . . 3

\section{List of Figures}

2 Reflection spectra of TM (solid blue) and TE (dashed red) mode. . . . . . . . . 5

3 Reflection spectra of TM (solid blue) and TE (dashed red) mode of the silica sublayer mirror with a $152 \mathrm{~nm}$ bandwidth. . . 6

4 Second design with a silicium sublayer. . . 7

5 Tolerance of the grating period : normalized bandwidth $\Delta \lambda / \lambda_{0}$ vs. grating period $\Lambda$ for a $R_{T M}$ threshold of $99.9 \%$ (black) and $99.99 \%$ (purple). The arrow indicates the design found by the optimization algorithm. ............ 8

\section{List of Tables}

1 Lengths and tolerances of the optimized mirror with a silica sublayer. . . . . . 5

2 Lengths and tolerances of the optimized mirror with a silicium sublayer. . . . . . 6 